\title{
An Epidemic Model for Tick-Borne Disease with Two Delays
}

\author{
Dan Li, Wanbiao Ma, and Zhichao Jiang \\ Department of Applied Mathematics, School of Mathematics and Physics, University of Science and Technology Beijing, \\ Beijing 100083, China \\ Correspondence should be addressed to Wanbiao Ma; wanbiao_ma@ustb.edu.cn
}

Received 23 September 2013; Accepted 18 November 2013

Academic Editor: Hui-Shen Shen

Copyright (C) 2013 Dan Li et al. This is an open access article distributed under the Creative Commons Attribution License, which permits unrestricted use, distribution, and reproduction in any medium, provided the original work is properly cited.

We have considered an epidemic model of a tick-borne infection which has nonviraemic transmission in addition to the viremic transmission. The basic reproduction number $\mathfrak{R}_{0}$, which is a threshold quantity for stability of equilibria, is calculated. If $\mathfrak{R}_{0} \leq 1$, then the infection-free equilibrium is globally asymptotically stable, and this is the only equilibrium. On the contrary, if $\Re_{0}>1$, then an infection equilibrium appears which is globally asymptotically stable, when one time delay is absent. By applying a permanence theorem for infinite dimensional systems, we obtain that the disease is always present when $\mathfrak{R}_{0}>1$.

\section{Introduction}

Vector-borne diseases are infectious diseases caused by viruses, bacteria, protozoa, or rickettsia which are primarily transmitted by disease-transmitting biological agents, called vectors, who carry the disease without getting it themselves. Vector-borne disease, in particular, tick-borne disease, such as Lyme disease, transmitted between humans by bloodfeeding ticks, have been big concerns for the public health in the world.

The dynamics of the tick vector are based on the biology of the tick Ixodes ricinus that transmits the virus that causes Louping ill. Louping ill is a tick-borne, zoonotic, viral disease that is most important in sheep and red grouse. This tick has a life cycle that develops from the egg through two immature stages (larva and nymph) to the adult stage. Each immature stage requires a blood meal from a suitable vertebrate host. The adult female requires a meal before producing eggs once and then dying. Adult females can only obtain a feed from large mammals, that is, hares and not grouse, whilst the immature stages will also feed on smaller warm-blooded vertebrates such as grouse chicks. Once a questing tick locates a host it generally feeds for several days. The tick life cycle usually takes 3 to 4 years but can be longer depending on host availability and climatic conditions; ticks need high relative humidity to survive [1].

Mathematical models have made considerable contributions to our understanding of tick-borne infections [1-8] or tick population dynamics [9-11]. Transmission from host to tick is generally believed to occur when ticks bite and feed on the blood of a viraemic host. However, in recent years it has become clear that pathogen transmission can occur through a number of other routes. For example, transmission between infected and uninfected ticks that cofeed on a host can occur in the absence of systemic infection [1]. Another route of infection for some tick-borne pathogens is through nonviraemic transmission. This occurs via the skin aided by tick saliva when a susceptible tick feeds close to an infected tick on a host that is not displaying high levels of viraemia in the blood [12]. Grouse chicks are known to eat ticks as part of their diet of invertebrates during the first three weeks of life. Gilbert et al. [13] suggest that the ingestion of infected ticks may be the main route of infection in wild grouse chicks; the authors consider the ingestion model; in this case, the virus persists over a greater range of grouse densities when ingestion is included. This is because it is now easier for the grouse to become infected with the inclusion of an extra route of infection via the oral route. In this paper, we consider the nonseasonal model; that is, the behavior of grouse and ticks was assumed constant over the course of a year. The non seasonal model can be analyzed analytically unlike the seasonal models. To investigate whether the incorporation of the seasonal biology of grouse alters the model predictions dramatically from the non seasonal model. In [13], they present a more realistic model which describes the chicks 
and adult grouse separately with chicks hatching only at a given time of the year. In this paper we build on the model by Norman et al. [1], which have proposed an epidemic dynamics of Louping ill virus in red grouse (the viremic host) and hares (the nonviremic host) in the following form:

$$
\begin{aligned}
\dot{G}_{s}(t)= & \left(a_{g}-s_{g} G_{s}(t)\right) G_{s}(t) \\
& -\beta_{1} T_{i}(t) G_{s}(t)-b_{g} G_{s}(t), \\
\dot{G}_{i}(t)= & \beta_{1} T_{i}(t) G_{s}(t)-\Gamma G_{i}(t), \\
\dot{G}_{z}(t)= & \gamma G_{i}(t)-b_{g} G_{z}(t), \\
\dot{T}_{s}(t)= & \left(a_{T}-s_{T} T_{s}(t)\right) T_{s}(t)-\beta_{2} T_{s}(t) G_{i}(t) \\
& -\theta H T_{s}(t) T_{i}(t)-\left(\beta_{3} H+b_{T}\right) T_{s}(t), \\
\dot{T}_{i}(t)= & \beta_{2} T_{s}(t) G_{i}(t)+\theta H T_{s}(t) T_{i}(t) \\
& -\left(\beta_{3} H+b_{T}\right) T_{i}(t) .
\end{aligned}
$$

The model considers two hosts, a viremic host that does not support adult ticks and a non-viremic host that supports all stages. The total viremic host population size at time $t$, given by $G(t)$, is partitioned into subclasses of individuals who are susceptible, infectious, and recovered, with sizes denoted by $G_{s}(t), G_{i}(t)$, and $G_{z}(t)$, respectively. Hares are assumed to be at a constant density $H . T_{s}(t)$ and $T_{i}(t)$ represent population density of susceptible ticks and infected ticks at any time $t$, with the total tick population, $T(t)=T_{s}(t)+T_{i}(t)$.

The rate of non-viremic transmission is assumed to be proportional to the number of hares present, the number of infected ticks present and the number of susceptible ticks present, and therefore takes the form $\theta H T_{i} T_{s}$, where $\theta$ is a measure of the probability of non-viremic transmission occurring. Furthermore, the viremic host population dies at a natural death rate $b_{g}$. The vertical transmission in the viremic host does not occur so that all newly recruited individuals are susceptible. The rate at which a grouse is bitten and infected by a tick $\beta_{1}$ can only involve the nymphal stage as adults do not generally bite grouse and the larvae are not infected. The rate at which nymphs bite grouse was estimated in [14] from tick counts of nymphs on deer and grouse as well as from model predictions; $\gamma$ is the rate at which infectious grouse become immune, and $\Gamma=\alpha+b_{g}+\gamma$ is the rate at which infectious grouse are lost from the system and is the sum of the death rate, due to the disease, the natural death rate, and the rate at which individuals become immune. For the ticks, $b_{T}$ is the per capita natural death rate. $\beta_{2}$ is the probability of an average tick biting a grouse and becoming infected per unit time, $\beta_{3}$ is the probability of an adult tick being female, biting a hare, and then going on to reproduce per unit time before dying and being lost from the system.

Norman et al. [1] consider the local stability of six biological relevant equilibria; this is done by determining the eigenvalues of the Jacobian evaluated at each equilibrium. The author chose the logistic growth function to describe the growth of grouse and ticks, obviously, it is more reasonable, but it increases the complexity of the algebraic analysis. To obtain global properties for model (1), we suppose that the host and tick population are recruited at rate that $\lambda$ and $s$. Note that the variables $G_{z}$ do not appear in the other equations of the above system. This allows us to consider the following fours dimensional system:

$$
\begin{gathered}
\dot{G}_{s}(t)=\lambda-\beta_{1} T_{i}(t) G_{s}(t)-b_{g} G_{s}(t), \\
\dot{G}_{i}(t)=\beta_{1} T_{i}(t) G_{s}(t)-\Gamma G_{i}(t), \\
\dot{T}_{s}(t)=s-\beta_{2} T_{s}(t) G_{i}(t) \\
\quad-\theta H T_{s}(t) T_{i}(t)-\left(\beta_{3} H+b_{T}\right) T_{s}(t), \\
\dot{T}_{i}(t)=\beta_{2} T_{s}(t) G_{i}(t) \\
+\theta H T_{s}(t) T_{i}(t)-\left(\beta_{3} H+b_{T}\right) T_{i}(t) .
\end{gathered}
$$

The incubation period for Louping ill is 6 to 18 days in sheep. Parenterally inoculated red grouse develop clinical signs in 2 to 8 days; the dynamic of tick feeding the incubation is effectively 12 months. We introduce two types of the delays that correspond to (i) the latent period in an infected host and (ii) the latency in a vector. We know that the number of infected hosts at time $t$ may depend on the number of susceptible hosts which is infected by the infected ticks at time $t-\tau$, for a time delay $\tau>0$, and $e^{-b_{g} \tau}$ is the survival rate. The number of infected ticks at time $t$ may depend on the number of susceptible ticks which is feeding on the infected hosts at time $t-\omega$, for a time delay $\omega>0$, and $e^{-b_{T} \omega}$ is the survival rate. Thus, the following model is proposed:

$$
\begin{aligned}
\dot{G}_{s}(t)= & \lambda-e^{-b_{g} \tau} \beta_{1} T_{i}(t-\tau) G_{s}(t)-b_{g} G_{s}(t), \\
\dot{G}_{i}(t)= & e^{-b_{g} \tau} \beta_{1} T_{i}(t-\tau) G_{s}(t)-\Gamma G_{i}(t), \\
\dot{T}_{s}(t)= & s-e^{-b_{T} \omega} \beta_{2} T_{s}(t) G_{i}(t-\omega) \\
& -\theta H T_{s}(t) T_{i}(t)-\left(\beta_{3} H+b_{T}\right) T_{s}(t), \\
\dot{T}_{i}(t)= & e^{-b_{T} \omega} \beta_{2} T_{s}(t) G_{i}(t-\omega) \\
& +\theta H T_{s}(t) T_{i}(t)-\left(\beta_{3} H+b_{T}\right) T_{i}(t) .
\end{aligned}
$$

Although the models incorporation of time delay have more real biological meaning, they are difficult to deal with it mathematically. However, the Lyapunov functional and the LaSalle-type theorem in [15] can provide a direct and effective method to establish global dynamical properties for the system of those nonlinear functional differential equations. Recently, Korobeinikov [16-18], and Huang et al. [19] have investigated the global dynamical properties of epidemiological models with and without delay by construc suitable Lyapunov functional methods. Inspired by the work in McCluskey [20], we construct a global Lyapunov functional and show that the disease-free equilibrium of system (3) is globally asymptotically stable if $\mathfrak{R}_{0} \leq 1$, and if $\mathfrak{R}_{0}>1$ the infection equilibrium is globally asymptotically stable when one delay is absent. Furthermore, by applying a permanence theorem by Smith and Zhao [21] for infinite dimensional 
systems (also see [22]), we obtain that the disease is always present when $\mathfrak{R}_{0}>1$. For its applications, we refer the reader to $[10,11,21-23]$.

The paper is organized as follows. In the next section, we analyze some basic mathematical properties (positivity and boundedness of solutions). In Section 3, we give a complete global stability analysis of the disease-free equilibrium. In Section 4, we are concerned with the global stability of the infection equilibrium with one delay. In Section 5, we show that the disease is endemic in the sense of permanence whenever $\mathfrak{R}_{0}>1$. A discussion of the mathematical results and of their biological implications is presented in Section 6 .

\section{Nonnegativity and Boundedness of Solutions}

Let $C=C\left([-\sigma, 0] ; R^{4}\right)$ be the Banach space of continuous functions from $[-\sigma, 0]$ to $R^{4}$ equipped with the sup-norm, where $\sigma=\max \{\tau, \omega\}$. The initial condition of (3) is given as

$$
\begin{gathered}
G_{s}(\theta)=\varphi_{1}(\theta), \quad G_{i}(\theta)=\varphi_{2}(\theta), \\
T_{s}(\theta)=\varphi_{3}(\theta), \quad T_{i}(\theta)=\varphi_{4}(\theta) \quad \theta \in[-\sigma, 0],
\end{gathered}
$$

where $\varphi=\left(\varphi_{1}, \varphi_{2}, \varphi_{3}, \varphi_{4}\right) \in C$ such that $\varphi_{j}(\theta) \geq 0(\sigma \leq \theta \leq$ $0, j=1,2,3,4)$.

The following result establishes the feasible region of the model and shows that the model is wellposed.

Theorem 1. Under initial conditions in (4), all solutions of system (3) are nonnegative on $[0,+\infty)$ and ultimately bounded.

Proof. First, we prove that $G_{s}(t)$ is positive for $t \geq 0$. Assuming the contrary and letting $t_{1}>0$ be the first time such that $G_{s}\left(t_{1}\right)=0$, by the first equation of system (3), we have $G_{s}^{\prime}\left(t_{1}\right)=\lambda>0$, and hence $G_{s}(t)<0$ for $t \in\left(t_{1}-\epsilon, t_{1}\right)$, where $\epsilon$ is an arbitrarily small positive constant. This leads to a contradiction. It follows that $G_{s}(t)$ is always positive. Using the same method, we have that $T_{s}(t)$ is always positive. Further, from the second and the forth equations in (3), we have, respectively,

$$
\begin{aligned}
G_{i}(t)= & G_{i}(0) e^{-\Gamma t} \\
& +e^{-b_{g} \tau} \beta_{1} \int_{0}^{t} T_{i}(\xi-\tau) G_{s}(\xi) e^{-\Gamma(t-\xi)} d \xi, \\
T_{i}(t)= & T_{i}(0) e^{-\int_{0}^{t}\left(\theta H T_{s}(\gamma)-\left(\beta_{3} H+b_{T}\right)\right) d \gamma}+e^{-b_{T} \omega} \beta_{2} \\
& \times \int_{0}^{t} T_{s}(\xi) G_{i}(\xi-\omega) e^{-\int_{\xi}^{t}\left(\theta H T_{s}(\gamma)-\left(\beta_{3} H+b_{T}\right)\right) d \gamma} d \xi .
\end{aligned}
$$

Using (4) we know that the $G_{i}(t)$ and $T_{i}(t)$ of (3) are positive for all $t \geq 0$.

Next we show that positive solutions of (3) are ultimately bounded for $t \geq 0$.
Define $F(t)=G_{s}(t)+G_{i}(t)+T_{s}(t)+T_{i}(t)$ and $d=$ $\min \left\{b_{g}, \beta_{3} H+b_{T}\right\}$. By non-negativity of the solution, it follows that

$$
\begin{aligned}
& F^{\prime}(t)= \lambda+s-\left(b_{g} G_{s}+\Gamma G_{i}+\left(\beta_{3} H+b_{T}\right)\right. \\
&\left.\times\left(T_{s}+T_{i}\right)\right) \\
& \leq \lambda+s-d F(t) .
\end{aligned}
$$

This implies that $F(t)$ is ultimately bounded, and so are $G_{s}(t)$, $G_{i}(t), T_{s}(t)$, and $T_{i}(t)$.

This completes the proof of Theorem 1.

The basic reproduction number [24] for system (3) is

$$
\mathfrak{R}_{0}=\frac{e^{-b_{g} \tau-b_{T} \omega} \lambda s \beta_{1} \beta_{2}}{\Gamma b_{g}\left(\beta_{3} H+b_{T}\right)^{2}}+\frac{s \theta H}{\left(\beta_{3} H+b_{T}\right)^{2}} .
$$

$\mathfrak{R}_{0}$ represents the average number of secondary infections that single infections host can generate in a totally susceptible population of hosts and ticks. The basic reproduction number consists of two terms representing two routes of infection. The tick would live for $1 /\left(\beta_{3} H+b_{T}\right)$ units of time and produces $\left(e^{-b_{g} \tau} \lambda \beta_{1}\right) / b_{g}\left(\beta_{3} H+b_{T}\right)$ infected grouse through feeding and $\theta s H /\left(\beta_{3} H+b_{T}\right)^{2}$ infected ticks through cofeeding. Each infected grouse lives for $1 / \Gamma$ units of time and produces $e^{-b_{T} \omega} \beta_{2} s / \Gamma\left(\beta_{3} H+b_{T}\right)$ infected ticks.

System (3) has always an infection-free equilibrium $E_{0}=$ $\left(G_{s}^{0}, 0, T_{s}^{0}, 0\right)$, that is, grouse and ticks both at their carrying capacity with no disease present, where $G_{s}^{0}=\lambda / b_{g}$, $T_{s}^{0}=s /\left(\beta_{3} H+b_{T}\right)$, and an infection equilibrium $E^{*}=$ $\left(G_{s}^{*}, G_{i}^{*}, T_{s}^{*}, T_{i}^{*}\right)$, when $\Re_{0}>1$, that is, ticks, grouse, and disease coexisting together, where $T_{i}^{*}$ is given by the following cubic:

$$
f\left(T_{i}^{*}\right)=A T_{i}^{* 2}+B T_{i}^{*}+C,
$$

where

$$
\begin{aligned}
A= & e^{-b_{g} \tau} \Gamma \theta H \beta_{1}\left(\beta_{3} H+b_{T}\right), \\
B=- & s \theta H \Gamma \beta_{1} e^{-b_{g} \tau} \\
& +e^{-b_{g} \tau-b_{T} \omega} \lambda \beta_{1} \beta_{2}\left(\beta_{3} H+b_{T}\right) \\
& +\Gamma\left(\beta_{3} H+b_{T}\right)\left(\theta H b_{g}+e^{-b_{g} \tau} \beta_{1}\left(\beta_{3} H+b_{T}\right)\right), \\
C= & -e^{-b_{g} \tau-b_{T} \omega} \lambda s \beta_{1} \beta_{2}-s \Gamma \theta H b_{g}+\Gamma b_{g}\left(\beta_{3} H+b_{T}\right)^{2} .
\end{aligned}
$$

Hence, we can write all of the densities in terms of the infectious tick density, $T_{i}^{*}$,

$$
\begin{aligned}
& G_{s}^{*}=\frac{\lambda}{e^{-b_{g} \tau} \beta_{1} T_{i}^{*}+b_{g}}, \\
& G_{i}^{*}=\frac{e^{-b_{g} \tau} \lambda \beta_{1} T_{i}^{*}}{\Gamma\left(e^{-b_{g} \tau} \beta_{1} T_{i}^{*}+b_{g}\right)}, \\
& T_{s}^{*}=\frac{s}{\beta_{3} H+b_{T}}-T_{i}^{*} .
\end{aligned}
$$




\section{Global Stability of the Infection-Free Equilibrium of Model (3)}

In this section, we would consider the global stability of the infection-free equilibrium. Next, we show that $\boldsymbol{R}_{0}$ determines the stability of the infection-free equilibrium.

Theorem 2. If $\mathfrak{R}_{0} \leq 1$, then the infection-free equilibrium $E_{0}$ is globally asymptotically stable for any time delay $\tau \geq 0$ and $\omega \geq 0$.

Proof. Consider a Lyapunov functional:

$$
V(t)=V_{1}(t)+V_{2}(t)+V_{3}(t),
$$

with

$$
\begin{aligned}
V_{1}(t)= & \frac{e^{-b_{T} \omega} \beta_{2} T_{s}^{0}}{\Gamma} \\
& \times\left(G_{s}(t)-G_{s}^{0}-G_{s}^{0} \ln \frac{G_{s}(t)}{G_{s}^{0}}\right)+\frac{e^{-b_{T} \omega} \beta_{2} T_{s}^{0}}{\Gamma} G_{i}(t) \\
& +\left(T_{s}(t)-T_{s}^{0}-T_{s}^{0} \ln \frac{T_{s}(t)}{t_{s}^{0}}\right)+T_{i}(t), \\
& V_{2}(t)=e^{-b_{T} \omega} \beta_{2} T_{s}^{0} \int_{t-\omega}^{t} G_{i}(\xi) d \xi \\
& V_{3}(t)=\frac{e^{-\left(b_{g} \tau+b_{T} \omega\right)} \beta_{1} \beta_{2} G_{s}^{0} T_{s}^{0}}{\Gamma} \int_{t-\tau}^{t} T_{i}(\xi) d \xi .
\end{aligned}
$$

Calculate the time derivative of $V$ :

$$
\begin{aligned}
\frac{d V}{d t}= & \frac{e^{-b_{T} \omega} \beta_{2} T_{s}^{0}}{\Gamma}\left(1-\frac{G_{s}^{0}}{G_{s}(t)}\right) G_{s}^{\prime}(t) \\
& +\frac{e^{-b_{T} \omega} \beta_{2} T_{s}^{0}}{\Gamma} G_{i}^{\prime}(t) \\
& +\left(1-\frac{T_{s}^{0}}{T_{s}(t)}\right) T_{s}^{\prime}(t)+T_{i}^{\prime}(t) \\
& +e^{-b_{T} \omega} \beta_{2} T_{s}^{0}\left(G_{i}(t)-G_{i}(t-\omega)\right) \\
& +\frac{e^{-\left(b_{g} \tau+b_{T} \omega\right)} \beta_{1} \beta_{2} G_{s}^{0} T_{s}^{0}}{\Gamma}\left(T_{i}(t)-T_{i}(t-\tau)\right) .
\end{aligned}
$$

Since $\lambda=b_{g} G_{s}^{0}, s=\left(\beta_{3} H+b_{T}\right) T_{s}^{0}$, it follows that

$$
\begin{aligned}
\frac{d V}{d t}= & \frac{\beta_{2} b_{g} G_{s}^{0} T_{s}^{0}}{\Gamma}\left(2-\frac{G_{s}^{0}}{G_{s}(t)}-\frac{G_{s}(t)}{G_{s}^{0}}\right) \\
& +\left(\beta_{3} H+b_{T}\right) T_{s}^{0}\left(2-\frac{T_{s}^{0}}{T_{s}(t)}-\frac{T_{s}(t)}{T_{s}^{0}}\right) \\
& +\left(\beta_{3} H+b_{T}\right)\left(\Re_{0}-1\right) T_{i}(t) .
\end{aligned}
$$

Obviously $\mathfrak{R}_{0} \leq 1$ ensures that $d V / d t \leq 0$. It is clear that $V \geq$ 0 and $V=0$ if and only if $G_{s}(t)=G_{s}^{0}, G_{i}(t)=0, T_{s}(t)=T_{s}^{0}$, and $T_{i}(t)=0$. Hence, it follows from the Lyapunov-LaSalle invariance principle that the infection-free equilibrium $E_{0}$ is globally asymptotically stable when $\mathfrak{R}_{0} \leq 1$.

This completes the proof of Theorem 2.

\section{Dynamical Analysis for the Infection Equilibrium}

System (3) has an infection equilibrium $E^{*}$ when $\Re_{0}>1$. We consider two situations: (a) $\tau=0, \omega \geq 0$ and (b) $\tau \geq 0, \omega=0$.

4.1. Global Stability of the Infection Equilibrium When $\tau=0$ and $\omega \geq 0$. In this subsection, the situations $\tau=0$ and $\omega \geq 0$ are considered.

Theorem 3. If $\mathfrak{R}_{0}>1$, then the infection equilibrium $E^{*}$ is globally asymptotically stable for $\tau=0$ and $\omega \geq 0$.

Proof. Define a Lyapunov functional:

$$
\begin{aligned}
U_{1}(t)= & \frac{e^{-b_{T} \omega} \beta_{2} T_{s}^{*} G_{i}^{*}}{\beta_{1} T_{i}^{*} G_{s}^{*}}\left(G_{s}(t)-G_{s}^{*}-G_{s}^{*} \ln \frac{G_{s}(t)}{G_{s}^{*}}\right) \\
& +\frac{e^{-b_{T} \omega} \beta_{2} T_{s}^{*} G_{i}^{*}}{\beta_{1} T_{i}^{*} G_{s}^{*}}\left(G_{i}(t)-G_{i}^{*}-G_{i}^{*} \ln \frac{G_{i}(t)}{G_{i}^{*}}\right) \\
& +\left(T_{s}(t)-T_{s}^{*}-T_{s}^{*} \ln \frac{T_{s}(t)}{T_{s}^{*}}\right) \\
& +\left(T_{i}(t)-T_{i}^{*}-T_{i}^{*} \ln \frac{T_{i}(t)}{T_{i}^{*}}\right) \\
& +e^{-b_{T} \omega} \beta_{2} T_{s}^{*} G_{i}^{*} U_{+}(t),
\end{aligned}
$$

where

$$
U_{+}(t)=\int_{0}^{\omega}\left(\frac{G_{i}(t-\theta)}{G_{i}^{*}}-1-\ln \frac{G_{i}(t-\theta)}{G_{i}^{*}}\right) d \theta .
$$

The derivatives of $U_{+}(t)$ are given by

$$
\begin{aligned}
\frac{d U_{+}(t)}{d t} & =\frac{d}{d t} \int_{0}^{\omega}\left(\frac{G_{i}(t-\theta)}{G_{i}^{*}}-1-\ln \frac{G_{i}(t-\theta)}{G_{i}^{*}}\right) d \theta \\
& =\int_{0}^{\omega} \frac{d}{d t}\left(\frac{G_{i}(t-\theta)}{G_{i}^{*}}-1-\ln \frac{G_{i}(t-\theta)}{G_{i}^{*}}\right) d \theta \\
& =-\int_{0}^{\omega} \frac{d}{d \theta}\left(\frac{G_{i}(t-\theta)}{G_{i}^{*}}-1-\ln \frac{G_{i}(t-\theta)}{G_{i}^{*}}\right) d \theta \\
& =\frac{G_{i}(t)}{G_{i}^{*}}-\frac{G_{i}(t-\omega)}{G_{i}^{*}}+\ln \frac{G_{i}(t-\omega)}{G_{i}(t)} .
\end{aligned}
$$


Hence, we obtain

$$
\begin{aligned}
& \frac{d U_{1}}{d t}=\frac{e^{-b_{T} \omega} \beta_{2} T_{s}^{*} G_{i}^{*}}{\beta_{1} T_{i}^{*} G_{s}^{*}}\left(1-\frac{G_{s}^{*}}{G_{s}(t)}\right) G_{s}^{\prime}(t) \\
& +\frac{e^{-b_{T} \omega} \beta_{2} T_{s}^{*} G_{i}^{*}}{\beta_{1} T_{i}^{*} S^{*}}\left(1-\frac{G_{i}^{*}}{G_{i}(t)}\right) G_{i}^{\prime}(t) \\
& +\left(1-\frac{T_{s}^{*}}{T_{s}(t)}\right) T_{s}^{\prime}(t)+\left(1-\frac{T_{i}^{*}}{T_{i}(t)}\right) T_{i}^{\prime}(t) \\
& +e^{-b_{T} \omega} \beta_{2} T_{s}^{*} G_{i}^{*} \frac{d U_{+}(t)}{d t} \\
& =\frac{e^{-b_{T} \omega} \beta_{2} T_{s}^{*} G_{i}^{*}}{\beta_{1} T_{i}^{*} G_{s}^{*}}\left(\lambda-\beta_{1} T_{i}(t) G_{s}(t)\right. \\
& \left.-b_{g} G_{s}(t)+\frac{G_{s}^{*}}{G_{s}(t)}\left(\lambda-\beta_{1} T_{i}(t) G_{s}(t)-b_{g} G_{s}(t)\right)\right) \\
& +\frac{e^{-b_{T} \omega} \beta_{2} T_{s}^{*} G_{i}^{*}}{\beta_{1} T_{i}^{*} G_{s}^{*}}\left(\beta_{1} T_{i}(t) G_{s}(t)-\Gamma G_{i}(t)\right. \\
& \left.+\frac{G_{i}^{*}}{G_{i}(t)}\left(\beta_{1} T_{i}(t) G_{s}(t)-\Gamma G_{i}(t)\right)\right) \\
& +\left(s-e^{-b_{T} \omega} \beta_{2} T_{s}(t) G_{i}(t-\omega)\right. \\
& -\theta H T_{s}(t) T_{i}(t)-\left(\beta_{3} H+b_{T}\right) T_{s}(t) \\
& +\frac{T_{s}^{*}}{T_{s}(t)}\left(s-e^{-b_{T} \omega} \beta_{2} T_{s}(t) G_{i}(t-\omega)\right. \\
& \left.\left.-\theta H T_{s}(t) T_{i}(t)-\left(\beta_{3} H+b_{T}\right) T_{s}(t)\right)\right) \\
& +\left(e^{-b_{T} \omega} \beta_{2} T_{s}(t) G_{i}(t-\omega)\right. \\
& +\theta H T_{s}(t) T_{i}(t)-\left(\beta_{3} H+b_{T}\right) T_{i}(t) \\
& +\frac{T_{i}^{*}}{T_{i}(t)}\left(e^{-b_{T} \omega} \beta_{2} T_{s}(t) G_{i}(t-\omega)\right. \\
& \left.\left.+\theta H T_{s}(t) T_{i}(t)-\left(\beta_{3} H+b_{T}\right) T_{i}(t)\right)\right) \\
& +e^{-b_{T} \omega} \beta_{2} T_{s}^{*} G_{i}^{*}\left(\frac{G_{i}(t)}{G_{i}^{*}}-\frac{G_{i}(t-\omega)}{G_{i}^{*}}\right. \\
& \left.+\ln \frac{G_{i}(t-\omega)}{G_{i}(t)}\right) .
\end{aligned}
$$

Since $\lambda=\beta_{1} T_{i}^{*} G_{s}^{*}+b_{g} G_{s}^{*}, s=e^{-b_{T} \omega} \beta_{2} T_{s}^{*} G_{i}^{*}-\theta H T_{s}^{*} T_{i}^{*}-$ $\left(\beta_{3} H+b_{T}\right) T_{s}^{*}$, and

$$
\begin{gathered}
\Gamma G_{i}^{*}=\beta_{1} T_{i}^{*} G_{s}^{*}, \\
\left(\beta_{3} H+b_{T}\right) T_{i}^{*}=e^{-b_{T} \omega} \beta_{2} T_{s}^{*} G_{i}^{*}+\theta H T_{s}^{*} T_{i}^{*},
\end{gathered}
$$

it follows that

$$
\begin{aligned}
\frac{d U_{1}}{d t}= & \frac{e^{-b_{T} \omega} \beta_{2} b_{g} G_{s}^{*} T_{s}^{*}}{\beta_{1} T_{i}^{*}} \\
& \times\left(2-\frac{G_{s}^{*}}{G_{s}(t)}-\frac{G_{s}(t)}{G_{s}^{*}}\right) \\
& +\theta H T_{i}^{*} T_{s}^{*}\left(2-\frac{T_{s}^{*}}{T_{s}(t)}-\frac{T_{s}(t)}{T_{s}^{*}}\right) \\
& +\left(\beta_{3} H+b_{T}\right) T_{s}^{*}\left(2-\frac{T_{s}^{*}}{T_{s}(t)}-\frac{T_{s}(t)}{T_{s}^{*}}\right) \\
& +e^{-b_{T} \omega} \beta_{2} T_{s}^{*} G_{i}^{*} \\
& \times\left(4-\frac{G_{s}^{*}}{G_{s}(t)}-\frac{G_{i}^{*} G_{s}(t) T_{i}(t)}{G_{s}^{*} T_{i}^{*} G_{i}(t)}\right. \\
& \left.\quad-\frac{T_{s}^{*}}{T_{s}(t)}-\frac{T_{i}^{*} T_{s}(t) G_{i}(t-\omega)}{T_{s}^{*} G_{i}^{*} T_{i}(t)}\right) .
\end{aligned}
$$

Using equality

$$
\begin{aligned}
\ln \frac{G_{i}(t-\omega)}{G_{i}(t)}= & \ln \frac{G_{s}^{*}}{G_{s}(t)}+\ln \frac{T_{s}^{*}}{T_{s}(t)} \\
& +\ln \frac{T_{i}^{*} T_{s}(t) G_{i}(t-\omega)}{T_{s}^{*} G_{i}^{*} T_{i}(t)}+\ln \frac{G_{i}^{*} G_{s}(t) T_{i}(t)}{G_{s}^{*} T_{i}^{*} G_{i}(t)},
\end{aligned}
$$

we obtain

$$
\begin{aligned}
\frac{d U_{1}}{d t}= & -b_{g} G_{s}^{*} \frac{1}{G_{s}(t)}\left(G_{s}(t)-G_{s}^{*}\right)^{2} \\
& -\theta H T_{i}^{*} T_{s}^{*} \frac{1}{T_{s}(t)}\left(T_{s}(t)-T_{s}^{*}\right)^{2} \\
& -\left(\beta_{3} H+b_{T}\right) T_{s}^{*} \frac{1}{T_{s}(t)}\left(T_{s}(t)-T_{s}^{*}\right)^{2} \\
& +e^{-b_{T} \omega} \beta_{2} T_{s}^{*} G_{i}^{*}\left(\Omega_{1}+\Omega_{2}+\Omega_{3}+\Omega_{4}\right),
\end{aligned}
$$

where

$$
\begin{aligned}
& \Omega_{1}=1-\frac{G_{s}^{*}}{G_{s}(t)}+\ln \frac{G_{s}^{*}}{G_{s}(t)}, \\
& \Omega_{2}=1-\frac{G_{i}^{*} G_{s}(t) T_{i}(t)}{G_{s}^{*} T_{i}^{*} G_{i}(t)}+\ln \frac{G_{i}^{*} G_{s}(t) T_{i}(t)}{G_{s}^{*} T_{i}^{*} G_{i}(t)}, \\
& \Omega_{3}=1-\frac{T_{s}^{*}}{T_{s}(t)}+\ln \frac{T_{s}^{*}}{T_{s}(t)}, \\
& \Omega_{4}=1-\frac{T_{i}^{*} T_{s}(t) G_{i}(t-\omega)}{T_{s}^{*} G_{i}^{*} T_{i}(t)}+\ln \frac{T_{i}^{*} T_{s}(t) G_{i}(t-\omega)}{T_{s}^{*} G_{i}^{*} T_{i}(t)} .
\end{aligned}
$$

Since the function

$$
g(t)=1-f(t)+\ln f(t)
$$


is always nonpositive for any function $f(t)>0$, and $g(t)=0$ if and only if $f(t)=1$. Therefore, we have $\Omega_{1}, \Omega_{2}, \Omega_{3}, \Omega_{4} \leq 0$.

It is easy to see that $d U_{1} / d t \leq 0$. It is clear that $d U_{1} / d t=0$ if and only if $G_{s}(t)=G_{s}^{*}, G_{i}(t)=G_{i}^{*}, T_{s}(t)=T_{s}^{*}, T_{i}(t)=T_{i}^{*}$. Hence, it is also known from stability theorem [25] that the infection equilibrium $E^{*}$ is globally asymptotically stable for any time delay $\omega \geq 0$ under the condition $\mathfrak{R}_{0}>1$.

This completes the proof of Theorem 3 .

4.2. Global Stability of the Infection Equilibrium When $\tau \geq 0$ and $\omega=0$. In this subsection, the situations $\tau \geq 0$ and $\omega=0$ are considered.

Theorem 4. If $\mathfrak{R}_{0}>1$, then the infection equilibrium $E^{*}$ is globally asymptotically stable for $\tau \geq 0$ and $\omega=0$.

Proof. Define a Lyapunov functional:

$$
\begin{aligned}
U_{2}(t)= & \frac{e^{b_{g} \tau} \beta_{2} T_{s}^{*} G_{i}^{*}}{\beta_{1} T_{i}^{*} G_{s}^{*}}\left(G_{s}(t)-G_{s}^{*}-G_{s}^{*} \ln \frac{G_{s}(t)}{G_{s}^{*}}\right) \\
& +\frac{e^{b_{g} \tau} \beta_{2} T_{s}^{*} G_{i}^{*}}{\beta_{1} T_{i}^{*} G_{s}^{*}}\left(G_{i}(t)-G_{i}^{*}-G_{i}^{*} \ln \frac{G_{i}(t)}{G_{i}^{*}}\right) \\
& +\left(T_{s}(t)-T_{s}^{*}-T_{s}^{*} \ln \frac{T_{s}(t)}{T_{s}^{*}}\right) \\
& +\left(T_{i}(t)-T_{i}^{*}-T_{i}^{*} \ln \frac{T_{i}(t)}{T_{i}^{*}}\right) \\
& +\beta_{2} T_{s}^{*} G_{i}^{*} U_{-}(t),
\end{aligned}
$$

where

$$
U_{-}(t)=\int_{0}^{\tau}\left(\frac{T_{i}(t-\theta)}{T_{i}^{*}}-1-\ln \frac{T_{i}(t-\theta)}{T_{i}^{*}}\right) d \theta .
$$

The derivatives of $U_{-}(t)$ are given by

$$
\begin{aligned}
\frac{d U_{-}(t)}{d t} & =\frac{d}{d t} \int_{0}^{\tau}\left(\frac{T_{i}(t-\theta)}{T_{i}^{*}}-1-\ln \frac{T_{i}(t-\theta)}{T_{i}^{*}}\right) d \theta \\
& =\int_{0}^{\tau} \frac{d}{d t}\left(\frac{T_{i}(t-\theta)}{T_{i}^{*}}-1-\ln \frac{T_{i}(t-\theta)}{T_{i}^{*}}\right) d \theta \\
& =-\int_{0}^{\tau} \frac{d}{d \theta}\left(\frac{T_{i}(t-\theta)}{T_{i}^{*}}-1-\ln \frac{T_{i}(t-\theta)}{T_{i}^{*}}\right) d \theta \\
& =\frac{T_{i}(t)}{T_{i}^{*}}-\frac{T_{i}(t-\tau)}{T_{i}^{*}}+\ln \frac{T_{i}(t-\tau)}{T_{i}(t)} .
\end{aligned}
$$

Hence, we obtain

$$
\begin{aligned}
\frac{d U_{2}}{d t}= & \frac{e^{b_{g} \tau} \beta_{2} T_{s}^{*} G_{i}^{*}}{\beta_{1} T_{i}^{*} G_{s}^{*}}\left(1-\frac{G_{s}^{*}}{G_{s}(t)}\right) G_{s}^{\prime}(t) \\
& +\frac{e^{b_{g} \tau} \beta_{2} T_{s}^{*} G_{i}^{*}}{\beta_{1} T_{i}^{*} S^{*}}\left(1-\frac{G_{i}^{*}}{G_{i}(t)}\right) G_{i}^{\prime}(t)
\end{aligned}
$$

$$
\begin{gathered}
+\left(1-\frac{T_{s}^{*}}{T_{s}(t)}\right) T_{s}^{\prime}(t) \\
+\left(1-\frac{T_{i}^{*}}{T_{i}(t)}\right) T_{i}^{\prime}(t)+\beta_{2} T_{s}^{*} G_{i}^{*} \frac{d U_{-}(t)}{d t} \\
=\frac{b_{g} \beta_{2} G_{s}^{*} T_{s}^{*} G_{i}^{*}}{\beta_{1} T_{i}^{*}}\left(2-\frac{G_{s}^{*}}{G_{s}(t)}-\frac{G_{s}(t)}{G_{s}^{*}}\right) \\
+\theta H T_{i}^{*} T_{s}^{*}\left(2-\frac{T_{s}^{*}}{T_{s}(t)}-\frac{T_{s}(t)}{T_{s}^{*}}\right) \\
+\left(\beta_{3} H+b_{T}\right) T_{s}^{*}\left(2-\frac{T_{s}^{*}}{T_{s}(t)}-\frac{T_{s}(t)}{T_{s}^{*}}\right) \\
+\beta_{2} T_{s}^{*} G_{i}^{*} \ln \frac{T_{i}(t-\tau)}{T_{i}(t)} \\
+\beta_{2} T_{s}^{*} G_{i}^{*}\left(4-\frac{G_{s}^{*}}{G_{s}(t)}-\frac{G_{i}^{*} G_{s}(t) T_{i}(t-\tau)}{G_{s}^{*} T_{i}^{*} G_{i}(t)}\right. \\
\left.-\frac{T_{s}^{*}}{T_{s}(t)}-\frac{T_{i}^{*} T_{s}(t) G_{i}(t)}{T_{s}^{*} G_{i}^{*} T_{i}(t)}\right) .
\end{gathered}
$$

Using equality

$$
\begin{aligned}
\ln \frac{T_{i}(t-\tau)}{T_{i}(t)}= & \ln \frac{G_{s}^{*}}{G_{s}(t)}+\ln \frac{T_{s}^{*}}{T_{s}(t)} \\
& +\ln \frac{T_{i}^{*} T_{s}(t) G_{i}(t)}{T_{s}^{*} G_{i}^{*} T_{i}(t)}+\ln \frac{G_{i}^{*} G_{s}(t) T_{i}(t-\tau)}{G_{s}^{*} T_{i}^{*} G_{i}(t)},
\end{aligned}
$$

we obtain

$$
\begin{aligned}
\frac{d U_{2}}{d t}= & -\frac{b_{g} \beta_{2} G_{s}^{*} T_{s}^{*} G_{i}^{*}}{\beta_{1} T_{i}^{*}} \frac{1}{G_{s}(t)}\left(G_{s}(t)-G_{s}^{*}\right)^{2} \\
& -\theta H T_{i}^{*} T_{s}^{*} \frac{1}{T_{s}(t)}\left(T_{s}(t)-T_{s}^{*}\right)^{2} \\
& -\left(\beta_{3} H+b_{T}\right) T_{s}^{*} \frac{1}{T_{s}(t)}\left(T_{s}(t)-T_{s}^{*}\right)^{2} \\
& +\beta_{2} T_{s}^{*} G_{i}^{*}\left(\Omega_{1}+\Omega_{5}+\Omega_{3}+\Omega_{6}\right),
\end{aligned}
$$

where

$$
\begin{gathered}
\Omega_{5}=1-\frac{G_{i}^{*} G_{s}(t) T_{i}(t-\tau)}{G_{s}^{*} T_{i}^{*} G_{i}(t)}+\ln \frac{G_{i}^{*} G_{s}(t) T_{i}(t-\tau)}{G_{s}^{*} T_{i}^{*} G_{i}(t)}, \\
\Omega_{6}=1-\frac{T_{i}^{*} T_{s}(t) G_{i}(t)}{T_{s}^{*} G_{i}^{*} T_{i}(t)}+\ln \frac{T_{i}^{*} T_{s}(t) G_{i}(t)}{T_{s}^{*} G_{i}^{*} T_{i}(t)} .
\end{gathered}
$$

Since the function

$$
g(t)=1-f(t)+\ln f(t)
$$

is always non-positive for any function $f(t)>0$, and $g(t)=0$ if and only if $f(t)=1$. Therefore, we have $\Omega_{1}, \Omega_{5}, \Omega_{3}, \Omega_{6} \leq 0$. 
It is easy to see that $d U_{2} / d t \leq 0$. It is clear that $d U_{2} / d t=0$ if and only if $G_{s}(t)=G_{s}^{*}, G_{i}(t)=G_{i}^{*}, T_{s}(t)=T_{s}^{*}, T_{i}(t)=T_{i}^{*}$. Hence, it is also known from stability theorem [25] that the infection equilibrium $E^{*}$ is globally asymptotically stable for any time delay $\tau \geq 0$ under the condition $\mathfrak{R}_{0}>1$.

This completes the proof of Theorem 4 .

\section{Permanence}

System (3) is said to be uniformly persistent if there exists a constant $\eta>0$ such that any solution $\left(G_{s}(t), G_{i}(t)\right.$, $\left.T_{s}(t), T_{i}(t)\right)$ of (3) satisfies

$$
\begin{array}{ll}
\liminf _{t \rightarrow \infty} G_{s}(t) \geq \eta, & \liminf _{t \rightarrow \infty} G_{i}(t) \geq \eta, \\
\liminf _{t \rightarrow \infty} T_{s}(t) \geq \eta, & \liminf _{t \rightarrow \infty} T_{i}(t) \geq \eta .
\end{array}
$$

Now we give a result on the uniform persistence of system (3). To proceed, we introduce the following notation and terminology. Denote by $P(t), t \geq 0$ the family of solution operators corresponding to (3). The $\omega$-limit set $\omega(x)$ of $x$ consists of $y \in X$ such that there exists a sequence $t_{n} \rightarrow \infty$ as $n \rightarrow \infty$ with $P\left(t_{n}\right) x \rightarrow y$ as $n \rightarrow \infty$.

Theorem 5. System (3) is uniformly persistent if it satisfies $\Re_{0}>1$.

Proof. Let

$$
\begin{gathered}
X^{0}=\left\{\phi \in X: \phi_{2}(0)>0, \phi_{4}(0)>0\right\}, \\
\partial X=X \backslash X^{0}=\left\{\phi \in X: \phi_{2}(0)=0 \text { or } \phi_{4}(0)=0\right\},
\end{gathered}
$$

which is relatively closed in $X$.

Now we prove that $X^{0}$ is positively invariant for $P(t)$. By the second and forth equations of (3), we have

$$
\begin{aligned}
& \frac{d G_{i}(t)}{d t} \geq-\Gamma G_{i}(t), \\
& \frac{d T_{i}(t)}{d t} \geq-\left(\beta_{3} H+b_{T}\right) T_{i}(t),
\end{aligned}
$$

$\forall t \geq 0$.

Since $G_{i}(0, \phi)=\phi_{2}(0)>0$ and $T_{i}(0, \phi)=\phi_{4}(0)>0$, it follows from (35) that

$$
\begin{array}{r}
G_{i}(t, \phi) \geq \phi_{2}(0) e^{-\Gamma t}, \\
T_{i}(t, \phi) \geq \phi_{4}(0) e^{-\left(\beta_{3} H+b_{T}\right) t}, \\
\forall t \geq 0 .
\end{array}
$$

Thus, $X^{0}$ is positively invariant for $P(t)$.

We set

$$
\begin{aligned}
M_{\partial}= & \{\phi \in X: \mathbf{Y}(\mathbf{t}) \phi \text { satisfies (3), } \\
& \mathbf{Y}(\mathbf{t}) \phi \in \partial X, \forall t \geq 0\} .
\end{aligned}
$$

We claim that

$$
M_{\partial}=\left\{\left(G_{s}, 0, T_{s}, 0\right)\right\} .
$$

Assuming $\mathbf{Y}(\mathbf{t}) \in M_{\partial}$, for all $t \geq 0$, it suffices to show that $G_{i}(t)=T_{i}(t)=0$, for all $t \geq 0$. If it is not true, then there exists $t_{0}>0$ such that either (a) $G_{i}\left(t_{0}\right)=0, T_{i}\left(t_{0}\right)>0$ or (b) $G_{i}\left(t_{0}\right)>0, T_{i}\left(t_{0}\right)=0$.

For case (a), from the forth equation of (3), we have

$$
\frac{d T_{i}(t)}{d t} \geq-\left(\beta_{3} H+b_{T}\right) T_{i}(t) .
$$

Since $T_{i}\left(t_{0}\right)>0$, we have

$$
T_{i}(t) \geq T_{i}\left(t_{0}\right) e^{-\left(\beta_{3} H+b_{T}\right)\left(t-t_{0}\right)}>0, \quad \forall t \geq t_{0} .
$$

By the invariant of $M_{\partial}$, we have $G_{i}(t) \equiv 0$, for all $t \geq 0$. Then from the second equation of (3), we have $T_{i}(t-\tau) G_{s}(t) \equiv 0$, for all $t \geq 0$.

From the first equation of (3), we obtain $\dot{G}_{s}(t)=\lambda-$ $b_{g} G_{s}(t)$, thus $\lim _{t \rightarrow+\infty} G_{s}(t)=\lambda / b_{g}$. By the invariant of $M_{\partial}$, we have $G_{s}(t)=\lambda / b_{g}$.

Then from the second equation of (3), we obtain $T_{i}(t-$ $\tau)=0$; by the invariant of $M_{\partial}$, we have $T_{i}(t) \equiv 0$, for all $t \geq 0$. From the first and third equations of (3), we obtain $\dot{T}_{s}(t)=$ $s-\left(\beta_{3} H+b_{T}\right) T_{s}(t)$; thus $\lim _{t \rightarrow+\infty} T_{s}(t)=s /\left(\beta_{3} H+b_{T}\right)$. By the invariant of $M_{\partial}$, we have $T_{s}(t)=s /\left(\beta_{3} H+b_{T}\right), \forall t \geq 0$, which contradicts the assumption that $\left(G_{s}(t), G_{i}(t), T_{s}(t), T_{i}(t)\right) \in$ $M_{\partial}$, for all $t \geq 0$. Similarly, we can obtain a contradiction for case (b). This proves the claim (38).

Let $A=\bigcap_{x \in A_{b}} \omega(x)$, where $A_{b}$ is the global attractor of $P(t)$ restricted to $\partial X$. We show that $A=E_{0}$. In fact, from $A \in M_{\partial}$ and the first and third equations of (3), we have $\lim _{t \rightarrow \infty} G_{s}(t)=G_{s}^{0}$ and $\lim _{t \rightarrow \infty} T_{s}(t)=T_{s}^{0}$. Thus, $E_{0}$ is the isolated invariant set in $X$.

Next, we show that $W^{s}\left(E_{0}\right) \cap X^{0}=\emptyset$. If this is not true, then there exists a solution $\left(G_{s_{t}}, G_{i_{t}}, T_{s_{t}}, T_{i_{t}}\right) \in X^{0}$ such that

$$
\begin{array}{ll}
\lim _{t \rightarrow \infty} G_{s}(t)=G_{s}^{0}, & \lim _{t \rightarrow \infty} G_{i}(t)=0, \\
\lim _{t \rightarrow \infty} T_{s}(t)=T_{s}^{0}, & \lim _{t \rightarrow \infty} T_{i}(t)=0 .
\end{array}
$$

For any sufficiently small constant $\varepsilon>0$, there exists a positive constant $T_{0}=T_{0}(\varepsilon)$ such that

$$
\begin{array}{ll}
G_{s}(t)>G_{s}^{0}-\varepsilon>0, & \forall t \geq T_{0}, \\
T_{s}(t)>T_{s}^{0}-\varepsilon>0, & \forall t \geq T_{0} .
\end{array}
$$

For the constant $\varepsilon$ given above, it follows from the second and forth equations of (3) that

$$
\begin{aligned}
\dot{G}_{i}(t) \geq & e^{-b_{g} \tau} \beta_{1} T_{i}(t-\tau) \\
& \times\left(G_{s}^{0}-\varepsilon\right)-\Gamma G_{i}(t), \\
\dot{T}_{i}(t) \geq & e^{-b_{T} \omega} \beta_{2}\left(T_{s}^{0}-\varepsilon\right) \\
& \times G_{i}(t-\omega)+\theta H\left(T_{s}^{0}-\varepsilon\right) T_{i}(t) \\
& -\left(\beta_{3} H+b_{T}\right) T_{i}(t) .
\end{aligned}
$$


If $G_{i}(t), T_{i}(t) \rightarrow 0$, as $t \rightarrow \infty$, then by a standard comparison argument and the nonnegativity, the solution $(x(t), y(t))$ of the following monotone system

$$
\begin{gathered}
\dot{x}(t)=e^{-b_{g} \tau} \beta_{1} y(t-\tau)\left(G_{s}^{0}-\varepsilon\right)-\Gamma x(t), \\
\dot{y}(t)=e^{-b_{T} \omega} \beta_{2}\left(T_{s}^{0}-\varepsilon\right) x(t-\omega) \\
+\theta H\left(T_{s}^{0}-\varepsilon\right) y(t) \\
-\left(\beta_{3} H+b_{T}\right) y(t)
\end{gathered}
$$

with the initial condition $x(t)=G_{i}(t), y(t)=T_{i}(t)$, for all $t \in$ $\left[t_{0}, t_{0}+T_{0}\right]$ converges to $(0,0)$ as well. Thus, $\lim _{t \rightarrow \infty} W(t)=0$, where $W(t)>0$ is defined by

$$
\begin{aligned}
W(t)= & \frac{e^{-b_{T} \omega} \beta_{2}\left(T_{s}^{0}-\varepsilon\right)}{\Gamma} x(t) \\
& +y(t)+e^{-b_{T} \omega} \beta_{2}\left(T_{s}^{0}-\varepsilon\right) \int_{t-\omega}^{t} x(\xi) d \xi \\
& +\frac{e^{-b_{g} \tau-b_{T} \omega} \beta_{1} \beta_{2}\left(G_{s}^{0}-\varepsilon\right)\left(T_{s}^{0}-\varepsilon\right)}{\Gamma} \int_{t-\tau}^{t} y(\xi) d \xi .
\end{aligned}
$$

Differentiating $W(t)$ with respect to time $t$ gives

$$
\begin{aligned}
& \left.\frac{d W(t)}{d t}\right|_{(44)} \\
& =\left[\theta H\left(T_{s}^{0}-\varepsilon\right)\right. \\
& \quad+\frac{e^{-b_{g} \tau-b_{T} \omega} \beta_{1} \beta_{2}\left(G_{s}^{0}-\varepsilon\right)\left(T_{s}^{0}-\varepsilon\right)}{\Gamma} \\
& \left.\quad-\left(\beta_{3} H+b_{T}\right)\right] y(t) .
\end{aligned}
$$

Because $\Re_{0}>1$, we have $\theta H\left(T_{s}^{0}-\varepsilon\right)+e^{-b_{g} \tau-b_{T} \omega} \beta_{1} \beta_{2}\left(G_{s}^{0}-\right.$ $\varepsilon)\left(T_{s}^{0}-\varepsilon\right) / \Gamma-\left(\beta_{3} H+b_{T}\right)>0$ for a sufficiently small $\varepsilon$. Therefore, $W(t)$ goes to either infinity or a positive number $t \rightarrow \infty$, which leads to a contradiction with $\lim _{t \rightarrow \infty} W(t)=$ 0 . Thus we have $W^{s}\left(E_{0}\right) \cap X^{0}=\emptyset$.

Define $p: X \rightarrow R_{+}$by

$$
p(\phi)=\min \left\{\phi_{2}(0), \phi_{4}(0)\right\}, \quad \forall \phi \in X .
$$

It is clear that $X^{0}=p^{-1}(0, \infty)$ and $\partial X=p^{-1}(0)$. Thus by [20, Theorem 3], we have

$$
\liminf _{t \rightarrow \infty}\left(G_{i}(t), T_{i}(t)\right) \geq\left(\eta_{0}, \eta_{0}\right)
$$

for some constant $\eta_{0}>0$. Let $\eta=\min \left\{\eta_{0}, \varepsilon\right\}$, where $\varepsilon$ is the constant such that $\liminf _{t \rightarrow \infty} G_{s}(t) \geq \varepsilon>0$ and $\liminf _{t \rightarrow \infty} T_{s}(t) \geq \varepsilon>0$. We showed that $\liminf _{t \rightarrow \infty} G_{s}(t) \geq$ $\eta, \liminf _{t \rightarrow \infty} G_{i}(t) \geq \eta, \liminf _{t \rightarrow \infty} T_{s}(t) \geq \eta$, and $\liminf _{t \rightarrow \infty} T_{i}(t) \geq \eta$.

This completes the proof of Theorem 5 .
TABLE 1: Parameter values used in the simulations shown in figures.

\begin{tabular}{lcc}
\hline Parameter & Value per month & Source \\
\hline$\lambda$ & varies & Assumed \\
$b_{g}$ & 0.087 & 20 \\
$\beta_{1}$ & 0.01 & 2 \\
$\beta_{2}$ & 0.02525 & 2 \\
$\alpha$ & 2.31 & 20 \\
$\gamma$ & 0.5775 & 2 \\
$s$ & varies & Assumed \\
$\theta$ & 0.00001 & 2 \\
$H$ & 10 & 2 \\
$b_{T}$ & 0.0277 & Unpublished date \\
$\beta_{3}$ & 0.0113 & 2 \\
\hline
\end{tabular}

\section{Numerical Simulations}

In this section, by using suitable numerical methods, we simulate the systems to illustrate the analytical results obtained in Sections 3-5. Moreover, we would verify the performance as shown in Section 5 and find that the system (3) is globally asymptotically stable when two delays are present simultaneously.

Table 1 shows the literature reported values for parameters which we used for the simulations. Besides the parameter value shown in Table 1 , we can find $\eta$ by using the equation $\eta=\alpha+\gamma+b_{g}$.

For system (3), we choose $\lambda=3, s=2, \tau=10$, and $\omega=$ 15 , and other values are shown in Table 1 . By computation, we obtain $R_{0}=0.0818<1$, and the infection equilibrium does not exist. By the analysis of Section 2, we know that the infection-free equilibrium $E_{0}=(34.4828,0,14.2146,0)$ is globally asymptotically stable (see Figure 1). Furthermore, we choose $\lambda=8, s=6, \tau=0$, and $\omega=15$, and other values are shown in Table 1. In Figure 2, we know that under the condition indicated in the Theorem 3 with $R_{0}=1.5645>1$ the infection equilibrium $E^{*}=(63.7684,1.324,38.421,4.15)$ will be globally asymptotically stable. In Figure 3, we know that under the condition indicated in the Theorem 4 with $R_{0}=1.8425>1$, the infection equilibrium $E^{*}=$ $(75.346,1.237,39.96,3.856)$ will be globally asymptotically stable with $\tau=3$ and $\omega=0$. In Figure 4 , we know that under the condition indicated in Theorem 5 with $R_{0}=1.1638>1$, the infection equilibrium $E^{*}=(68.72,1.0472,39.456,4.385)$ will be globally asymptotically stable with $\tau=5$ and $\omega=10$.

\section{Conclusion}

In the present paper, we investigate an epidemic model for tick-borne disease with non-viraemic transmission and two time delays. By constructing Lyapunov functionals, we prove that the infection-free equilibrium $E_{0}$ of system (3) is globally asymptotically stable when $\mathfrak{R}_{0} \leq 1$. If $\mathfrak{R}_{0}>1$ then the infection equilibrium $E^{*}$ is globally asymptotically stable when one delay is absent. By applying the persistence theory for infinite dimensional systems, we obtain that the populations of system (3) can coexist permanently if $\mathfrak{R}_{0}>1$. 


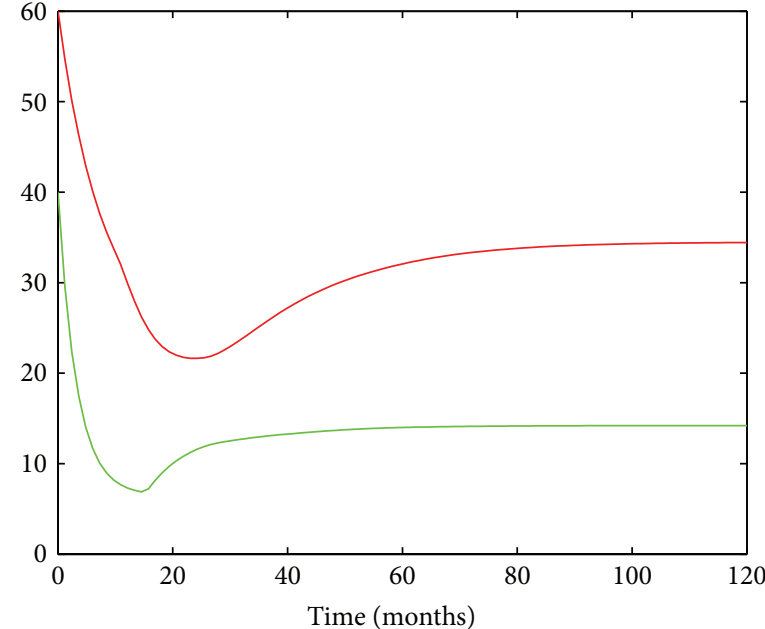

$-G_{s}(t)$

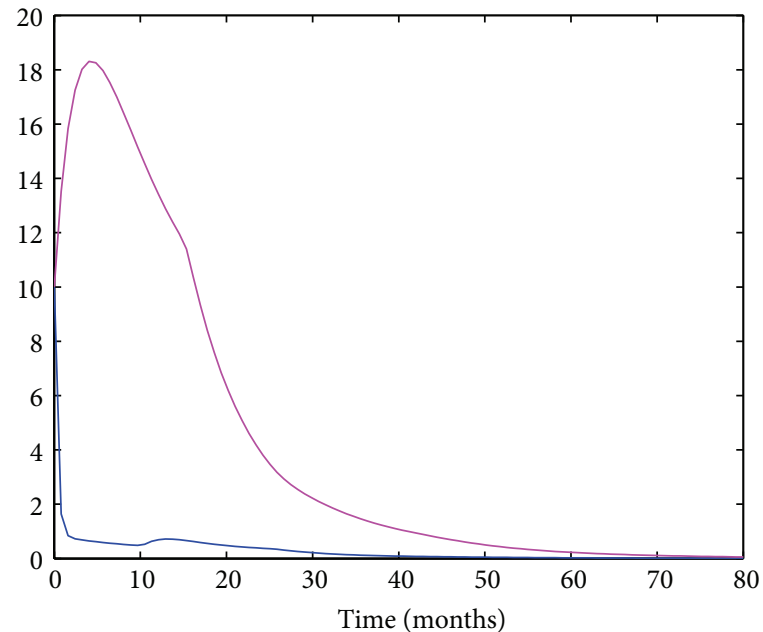

$-G_{i}(t)$

$-T_{i}(t)$

(a)

(b)

FIGURE 1: The infection-free equilibrium $E_{0}$ of system (3) is globally asymptotically stable when $\tau=10, \omega=15$, and $\mathfrak{R}_{0} \leq 1$. The initial functions are $(60,10,40$, and 10$)$.

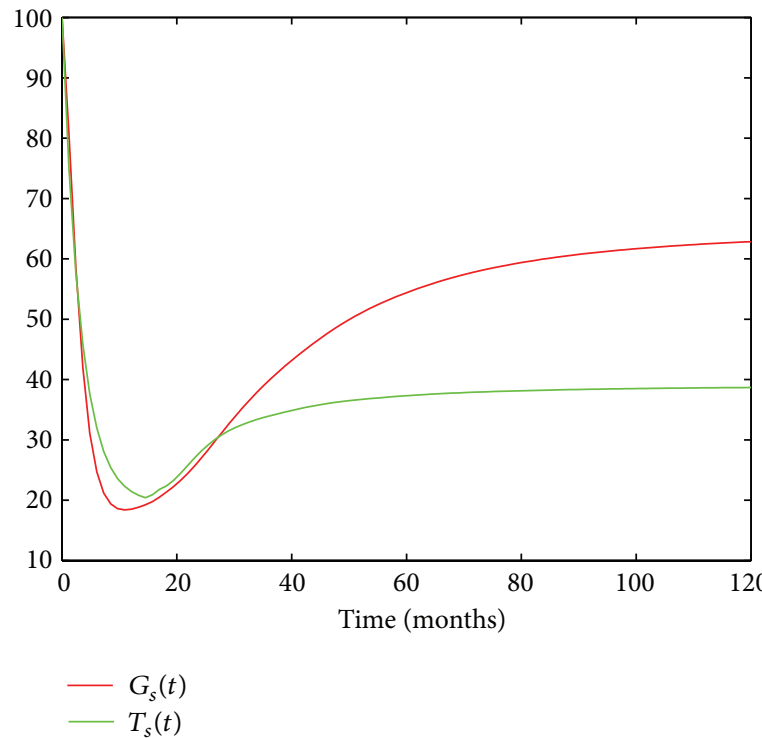

(a)

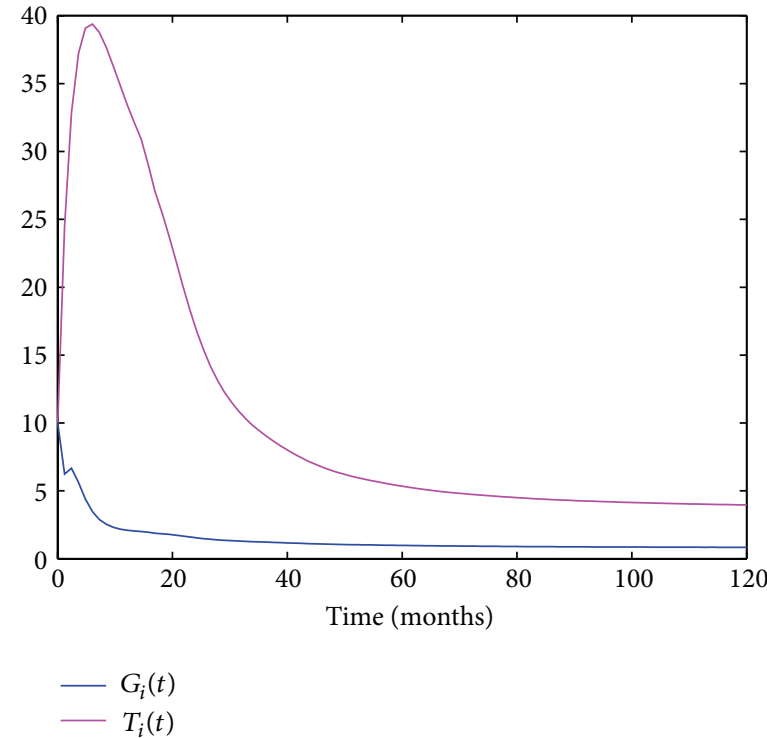

(b)

FIGURE 2: The infection equilibrium $E^{*}$ of system (3) is asymptotically stable when $\tau=0, \omega=15$, and $\mathfrak{R}_{0}>1$. The initial functions are $(100,10,100$, and 10$)$.

The reproductive number plays a crucial role for epidemic dynamics. Considering the basic reproductive number $\mathfrak{R}_{0}=$ $e^{-b_{g} \tau-b_{T} \omega} \lambda s \beta_{1} \beta_{2} / \Gamma b_{g}\left(\beta_{3} H+b_{T}\right)^{2}+s \theta H /\left(\beta_{3} H+b_{T}\right)^{2}$ as a function of $\tau$ and $\omega$, we can find that it is decreasing in $\tau$ and $\omega$ and it tends to 0 if the time delay $\tau$ or $\omega$ tends to 1. Therefore, the basic reproductive number can be reduced by increasing the intracellular delay. This may provide an insight for developing vaccination and regular treatment with acaricides that try to increase the intracellular delay.
It is interesting to consider the global stability of the infection equilibrium $E^{*}$ with two time delays; from the numerical simulations, it is shown that the infection equilibrium $E^{*}$ is globally asymptotically stable when $\Re_{0}>1$; we leave this as a future work.

The nonseasonal model can be analysed analytically unlike the seasonal models. The non seasonal model is also simpler and quicker to implement in Mathematica. However, the effect of the seasonal behavior of ticks will be explored 


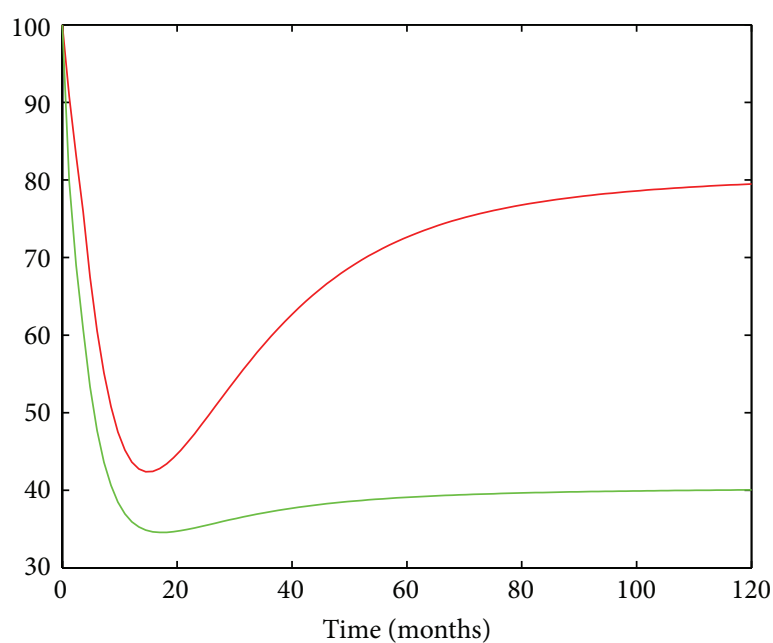

$$
\begin{array}{r}
-G_{s}(t) \\
-T_{s}(t)
\end{array}
$$

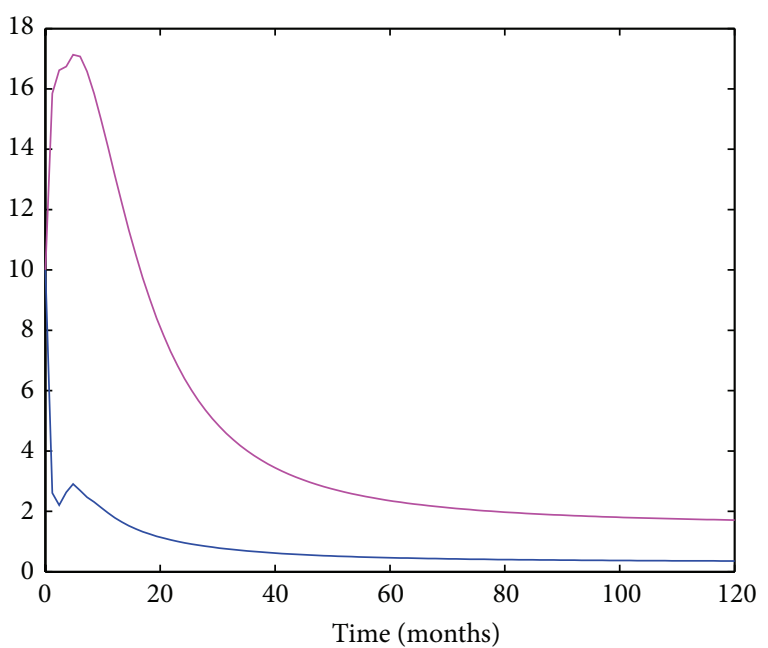

$-G_{i}(t)$
$-T_{i}(t)$

(a)

(b)

Figure 3: The infection equilibrium $E^{*}$ of system (3) is asymptotically stable when $\tau=3, \omega=0$, and $\mathfrak{R}_{0}>1$. The initial functions are $(100,10,100$, and 10$)$.
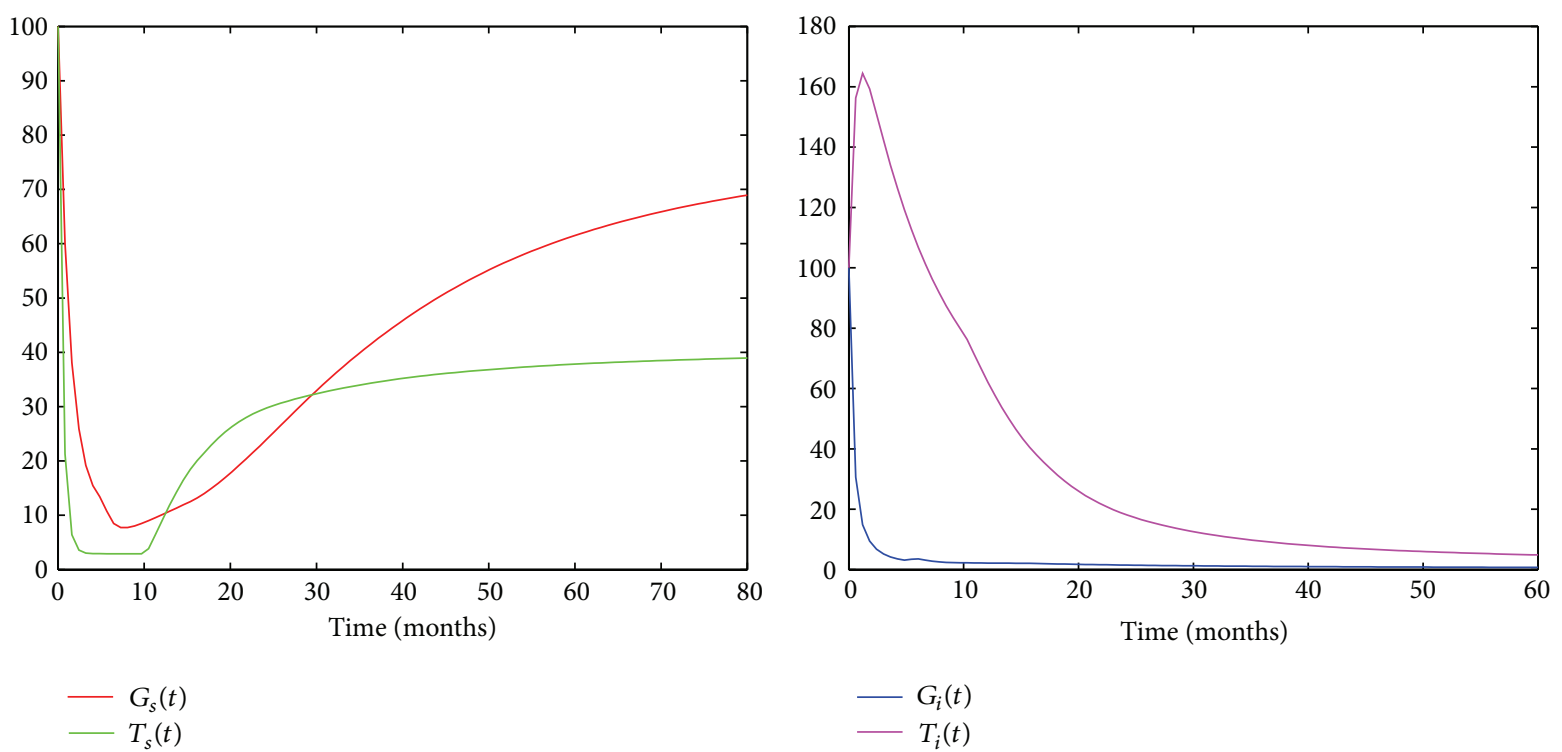

(a)

(b)

FIgURE 4: The infection equilibrium $E^{*}$ of system (3) is asymptotically stable when $\tau=5, \omega=10$, and $\Re_{0}>1$. The initial functions are $(100,10,100$, and 10$)$.

before deciding whether any form of seasonality will be incorporated in future work.

\section{Acknowledgment}

The research was partially supported by the Funds of USTB and NNSF of China (11071013).

\section{References}

[1] R. Norman, D. Ross, M. K. Laurenson, and P. J. Hudson, "The role of non-viraemic transmission on the persistence and dynamics of a tick borne virus-Louping ill in red grouse
(Lagopus lagopus scoticus) and mountain hares (Lepus timidus)," Journal of Mathematical Biology, vol. 48, no. 2, pp. 119-134, 2004.

[2] R. Norman, R. G. Bowers, M. Begon, and P. J. Hudson, "Persistence of tick-borne virus in the presence of multiple host species: tick reservoirs and parasite mediated competition," Journal of Theoretical Biology, vol. 200, no. 1, pp. 111-118, 1999.

[3] T. Caraco, G. Gardner, W. Maniatty, E. Deelman, and B. K. Szymanski, "Lyme disease: self-regulation and pathogen invasion," Journal of Theoretical Biology, vol. 193, no. 4, pp. 561$575,1998$.

[4] R. Rosà, A. Pugliese, R. Norman, and P. J. Hudson, "Thresholds for disease persistence in models for tick-borne infections 
including non-viraemic transmission, extended feeding and tick aggregation," Journal of Theoretical Biology, vol. 224, no. 3, pp. 359-376, 2003.

[5] S. Sandberg, T. E. Awerbuch, and A. Spielman, "A comprehensive multiple matrix model representing the life cycle of the tick that transmits the agent of Lyme disease," Journal of Theoretical Biology, vol. 157, no. 2, pp. 203-220, 1992.

[6] T. Awerbuch-Friedlander, R. Levins, and M. Predescu, "The role of seasonality in the dynamics of deer tick populations," Bulletin of Mathematical Biology, vol. 67, no. 3, pp. 467-486, 2005.

[7] P. J. Hudson, R. Norman, M. K. Laurenson et al., "Persistence and transmission of tick-borne viruses: ixodes ricinus and louping-ill virus in red grouse populations," Parasitology, vol. 111, pp. S49-S58, 1995.

[8] P. J. Hudson, "Grouse in space and time: the population biology of a managed gamebird," The report of the Game conservancys Scottish Grouse Research project and North of England Grouse Research project. Game Conservancy Trust Fordingbridge, 1992.

[9] H. Zhang and L. Chen, "A model for two species with stage structure and feedback control," International Journal of Biomathematics, vol. 1, no. 3, pp. 267-286, 2008.

[10] X. Song and H. Guo, "Global stability of a stage-structured predator-prey system," International Journal of Biomathematics, vol. 1, no. 3, pp. 313-326, 2008.

[11] H. G. Mwambi, J. Baumgartner, and K. P. Hadeler, "Ticks and tick-borne diseases: a vector-host interaction model for the brown ear tick (Rhipicephalus appendiculatus)," Statistical Methods in Medical Research, vol. 9, no. 3, pp. 279-301, 2000.

[12] M. Labuda, L. D. Jones, T. Williams, V. Danielova, and P. A. Nuttall, "Efficient transmission of tick-borne encephalitis virus between cofeeding ticks," Journal of Medical Entomology, vol. 30, no. 1, pp. 295-299, 1993.

[13] L. Gilbert, L. D. Jones, M. K. Laurenson, E. A. Gould, H. W. Reid, and P. J. Hudson, "Ticks need not bite their red grouse hosts to infect them with louping ill virus," Proceedings of the Royal Society B, vol. 271, no. 4, pp. S202-S205, 2004.

[14] L. Gilbert, R. Norman, K. M. Laurenson, H. W. Reid, and P. J. Hudson, "Disease persistence and apparent competition in a three-host community: an empirical and analytical study of large-scale, wild populations," Journal of Animal Ecology, vol. 70, no. 6, pp. 1053-1061, 2001.

[15] Y. Kuang, Delay Differential Equations with Applications in Population Dynamics, vol. 191 of Mathematics in Science and Engineering, Academic Press, Boston, Mass, USA, 1993.

[16] A. Korobeinikov, "Lyapunov functions and global properties for SEIR and SEIS epidemic models," Mathematical Medicine and Biology, vol. 21, no. 2, pp. 75-83, 2004.

[17] A. Korobeinikov and P. K. Maini, "A Lyapunov function and global properties for SIR and SEIR epidemiological models with nonlinear incidence," Mathematical Biosciences and Engineering, vol. 1, no. 1, pp. 57-60, 2004.

[18] A. Korobeinikov, "Global properties of infectious disease models with nonlinear incidence," Bulletin of Mathematical Biology, vol. 69, no. 6, pp. 1871-1886, 2007.

[19] G. Huang, Y. Takeuchi, W. Ma, and D. Wei, "Global stability for delay SIR and SEIR epidemic models with nonlinear incidence rate," Bulletin of Mathematical Biology, vol. 72, no. 5, pp. 11921207, 2010.

[20] C. C. McCluskey, "Complete global stability for an SIR epidemic model with delay-distributed or discrete," Nonlinear Analysis: Real World Applications, vol. 11, no. 1, pp. 55-59, 2010.
[21] H. L. Smith and X.-Q. Zhao, "Robust persistence for semidynamical systems," Nonlinear Analysis: Theory, Methods \& Applications, vol. 47, no. 9, pp. 6169-6179, 2001.

[22] X.-Q. Zhao, Dynamical Systems in Population Biology, CMS Books in Mathematics/Ouvrages de Mathématiques de la SMC, 16, Springer, New York, NY, USA, 2003.

[23] H. R. Thieme, "Persistence under relaxed point-dissipativity (with application to an endemic model)," SIAM Journal on Mathematical Analysis, vol. 24, no. 2, pp. 407-435, 1993.

[24] O. Diekmann, J. A. P. Heesterbeek, and J. A. J. Metz, "On the definition and the computation of the basic reproduction ratio $R_{0}$ in models for infectious diseases in heterogeneous populations," Journal of Mathematical Biology, vol. 28, no. 4, pp. 365-382, 1990.

[25] J. K. Hale and S. M. Verduyn Lunel, Introduction to FunctionalDifferential Equations, vol. 99 of Applied Mathematical Sciences, Springer, New York, NY, USA, 1993. 


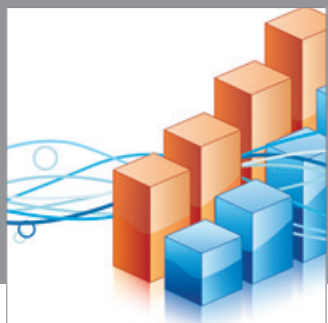

Advances in

Operations Research

mansans

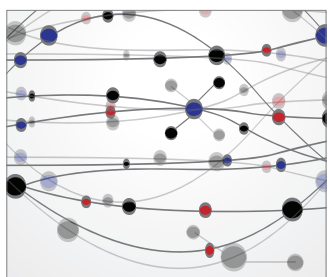

The Scientific World Journal
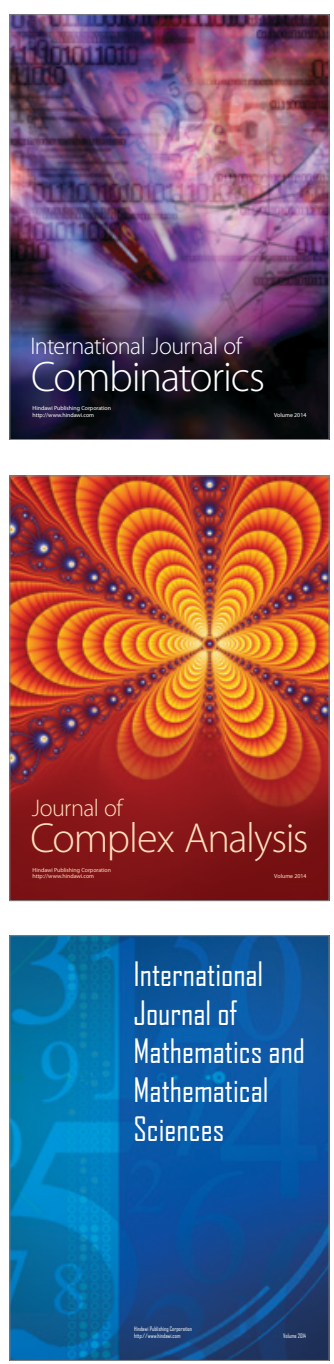
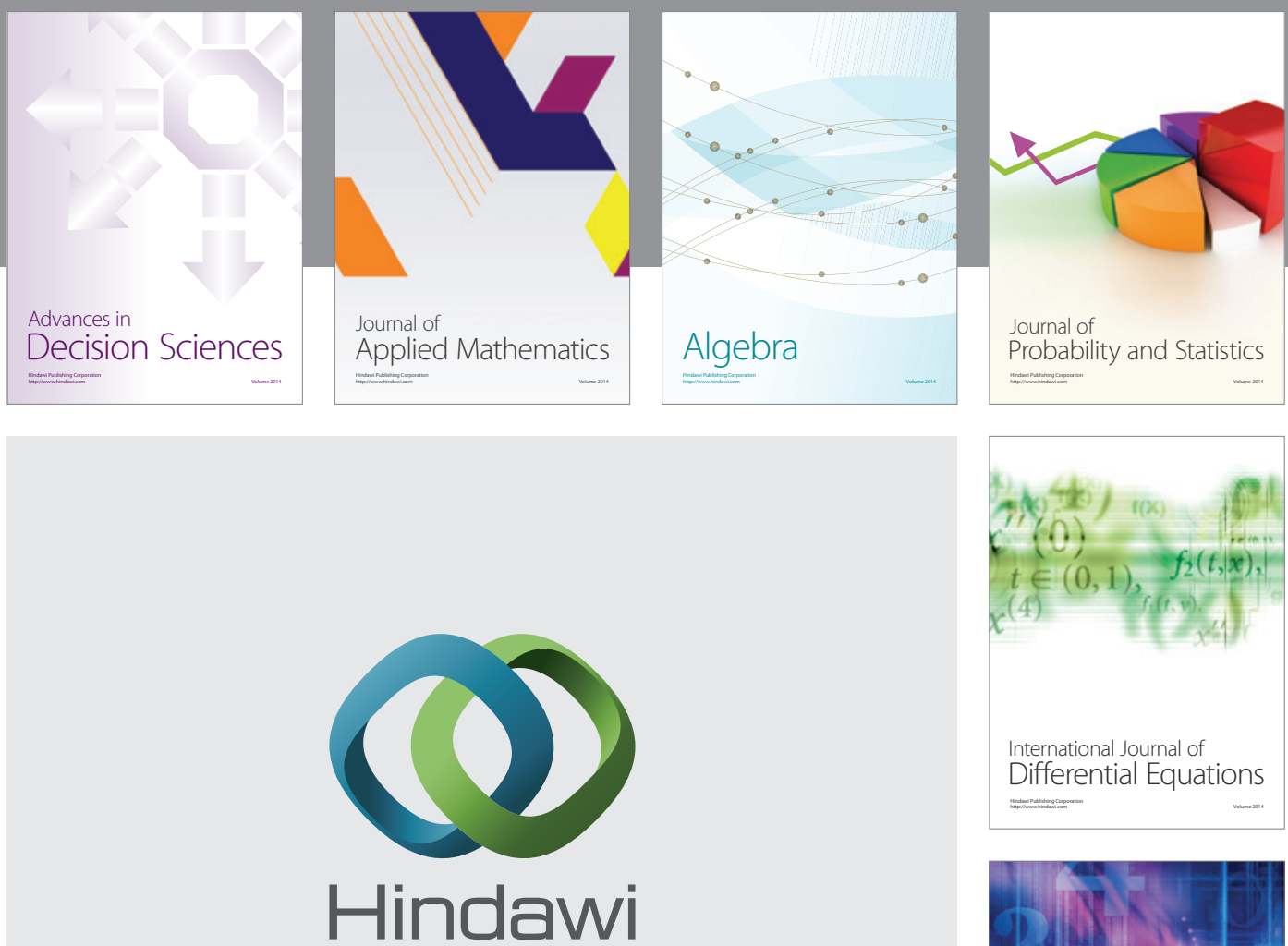

Submit your manuscripts at http://www.hindawi.com
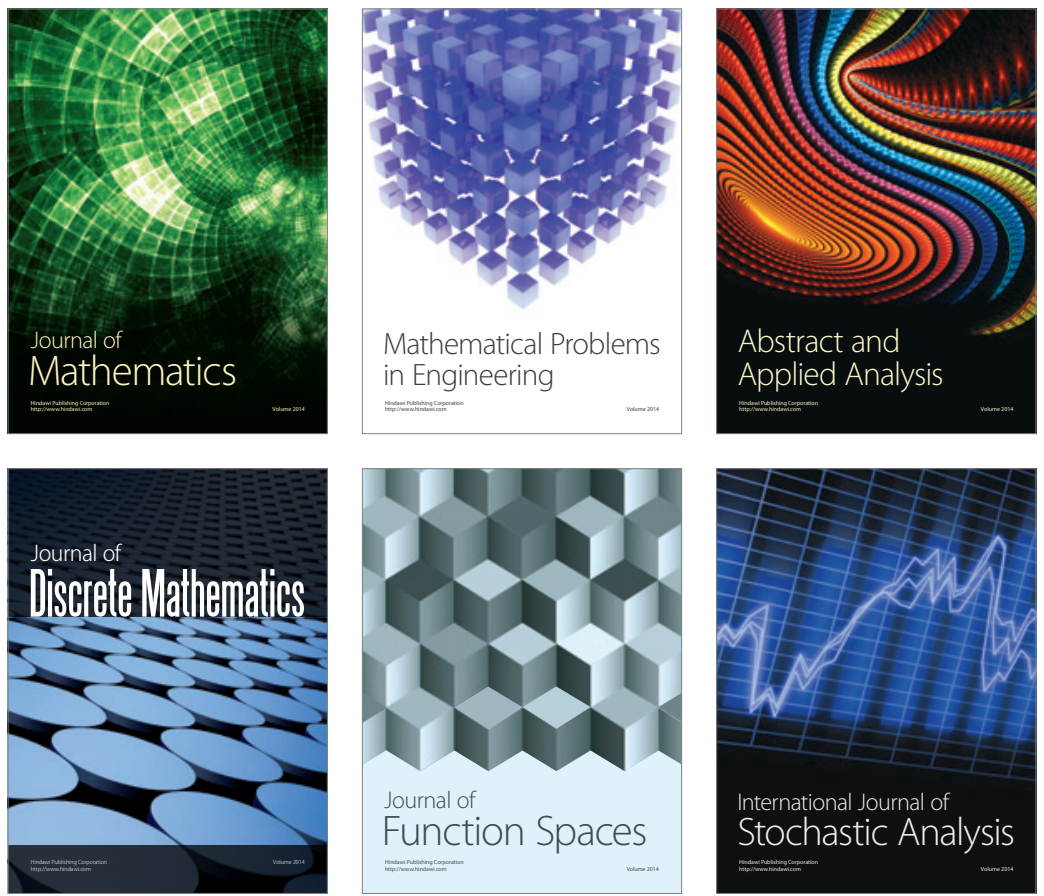

Journal of

Function Spaces

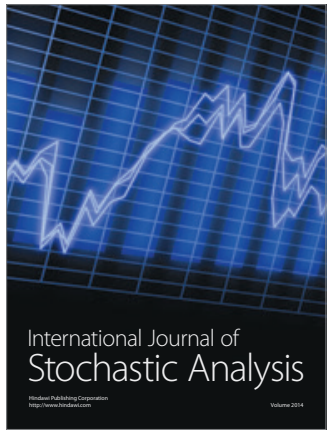

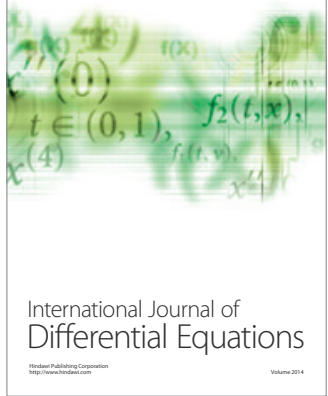
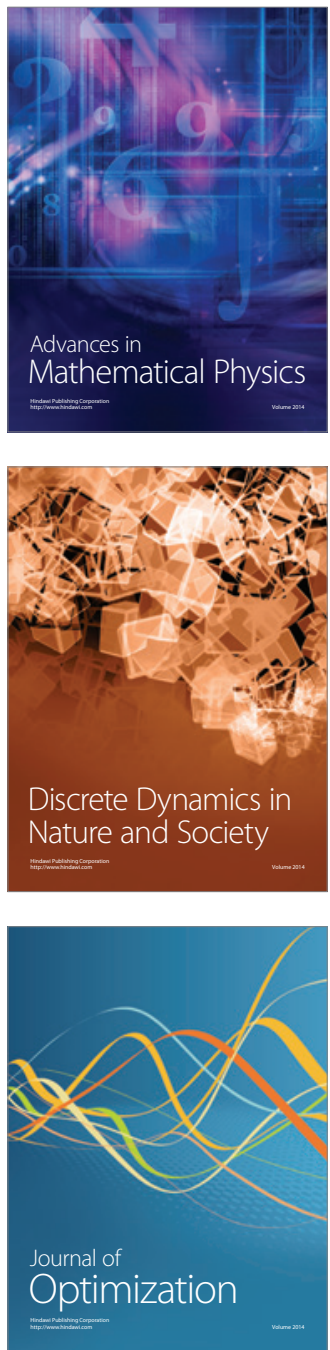